\title{
Escherichia coli endA deletion strain for use in two-hybrid shuttle vector selection
}

\author{
Malachi Griffith and R. Daniel Gietz \\ University of Manitoba, Winnipeg, MB, Canada
}

BioTechniques 35:272-278 (August 2003)

Escherichia coli has been used as the host organism for DNA cloning since its inception (1). As recombinant DNA technology evolves, novel bacterial strains are needed to complement new cloning strategies. In 1989, Fields and Song (2) developed the yeast twohybrid system. This system uses a pair of yeast- $E$. coli shuttle vectors to detect protein-protein interactions. Plasmids that contain putative interacting proteins are identified in yeast and shuttled back into $E$. coli for further analysis.

Certain yeast genes can complement specific mutations in $E$. coli (3). The $E$. coli strain, $\mathrm{KC} 8$, contains $p y r F$, leuB, $\operatorname{trp} C$, and his $B$ mutations that allow the selection of the Saccharomyces cerevisiae URA3, LEU2, TRP1, and HIS3 genes, respectively (4). However, KC8 does not produce high-quality plasmid DNA preparations due to the lack of an end $A$ gene mutation, a nonsequencespecific endonuclease. To overcome this problem, we have produced a new E. coli strain, MG7 $\alpha$, which contains deletion alleles for each of the $p y r F$, leuB, $\operatorname{trp} C$, his $B$, and end $A$ genes. MG7 $\alpha$ has some distinct advantages over KC8. In particular, it is sensitive to kanamycin, ampicillin, chloramphenicol, and tetracyclin. In addition, it provides $\alpha$-complementation of the $\beta$ galactosidase gene. Finally, MG7 $\alpha$ contains an endA deletion mutation. The end $A$ deletion allows the production of high-quality plasmid DNA using standard methods. Yeast-E. coli shuttle vectors containing any of the URA3, $L E U 2, T R P 1$, and HIS3 genes can be selected and identified in this strain.

The mutations were generated using the one-step inactivation of chromosomal genes method developed by Datsenko and Wanner (5). Bacteria were maintained on LB agar medium, and the cultures were grown in LB broth (Unless otherwise stated, all chemicals, media, and antibiotics are from SigmaAldrich Research Reagents, Oakville, ON, Canada). SOC broth (6) was used for the recovery of bacteria after electroporation. Kanamycin- and ampicillin-resistant transformants were selected on LB plates containing 25 $\mu \mathrm{g} / \mathrm{mL}$ kanamycin and $100 \mu \mathrm{g} / \mathrm{mL}$ ampicillin, respectively. M9 minimal medium (6) lacking uracil, leucine, tryptophan, or histidine was used to test the auxotrophic phenotype of putative mutants. A $0.2 \%(\mathrm{w} / \mathrm{v})$ final concentration of arabinose was used for the induction of the red recombinase system (5). PCR primers were produced and desalted by Canadian Life Technologies (Burlington, ON, Canada). The restriction enzyme DpnI was purchased from New England Biolabs (Mississauga, ON, Canada), and the Taq DNA polymerase was from Canadian Life Technologies. Plasmid DNA was extracted essentially as previously described (7). PCR comprised a 5-min initial denaturation at $94^{\circ} \mathrm{C}, 35$ cycles of 1 min denaturation at $94^{\circ} \mathrm{C}, 1 \mathrm{~min}$ annealing at $58^{\circ} \mathrm{C}$, and 1 min extension at $68^{\circ} \mathrm{C}$, followed by a final extension step for $7 \mathrm{~min}$ at $68^{\circ} \mathrm{C}$. The reaction conditions consisted of $0.2 \mathrm{mM}$ dNTPs, $1 \mu \mathrm{M}$ each primer, $0.25 \mu \mathrm{L} \mathrm{Taq}$ DNA polymerase, and approximately $50 \mathrm{ng}$ template DNA per $50 \mu \mathrm{L}$ reaction. PCR products were $D p n I$-treated to remove intact template plasmid molecules before being electrophoresed and gel purified, as previously described (8).

The $E$. coli strain DH5 $\alpha$ was used as the parental strain to produce MG7 $\alpha$. The E. coli strain KC8 was used as a benchmark strain for phenotypic testing. Table 1 summarizes the genotype of each strain.
The plasmids pKD46, pKD4, and pCP20 (5) were obtained from the $E$. coli Genetic Stock Center (http:// cgsc.biology.yale.edu/) and used in the process of gene disruption. Plasmids YEplac112 (URA3), YEplac181 (LEU2), YEplac195 (TRP1) (9), and pRS303 (HIS3) (10) were used to test for the complementation of the bacterial auxotrophic mutations.

An overnight culture of bacteria was made electrocompetent and transformed according to the method of Dower et al. (11). The cells were electroporated using a Gene Pulser ${ }^{\mathrm{TM}}$ (BioRad Laboratories, Hercules, CA, USA) at $1.25 \mathrm{kV}, 25 \mu \mathrm{F}$, with a $400 \Omega$ resistor in parallel with the sample chamber. To prepare the cells for efficient gene disruption, transformants containing the red helper plasmid (pKD46) were grown at $30^{\circ} \mathrm{C}$ in LB broth containing ampicillin $(50 \mu \mathrm{g} / \mathrm{mL})$ and arabinose for approximately $3 \mathrm{~h}$. These cells were transformed by electroporation (11) with 50-100 ng PCR-generated genedisruption cassette. The electroporated cells were immediately suspended in 1 $\mathrm{mL}$ SOC broth and incubated for $1 \mathrm{~h}$. Of the $1 \mathrm{~mL}, 500 \mu \mathrm{L}$ were spread onto kanamycin plates immediately after incubation, and the second $500 \mu \mathrm{L}$ were spread after standing overnight at room temperature. All colonies selected on kanamycin plates were maintained on LB agar plates without antibiotics. The auxotrophic phenotype of the mutants was tested by replica plating onto the appropriate M9 minimal media plates.

The FLP recombinase helper plasmid (pCP20) was used to remove the resistance marker after the successful identification of the target mutation. The plasmid was electroporated into electrocompetent cells prepared from a specific mutant strain. The resulting ampicillinresistant transformants were selected at $30^{\circ} \mathrm{C}$, which facilitated the removal of the antibiotic marker. The colonies were then streaked onto LB plates without antibiotic and grown overnight at $43^{\circ} \mathrm{C}$ to cure the strain of the pCP20 plasmid. The resulting colonies were tested for antibiotic resistances, and in most cases, the bacteria had become kanamycinand ampicillin-sensitive.

Lysates from each bacterial strain were prepared from overnight cultures grown in LB broth. The cells were har- 
Table 1. Genotype of Escherichia coli Bacterial Strains

\begin{tabular}{|c|c|}
\hline Strain & Genotype \\
\hline $\mathrm{DH} 5 \alpha(16)$ & $\begin{array}{l}F^{-} \text {, endA1, hsdR17 }\left(\mathrm{r}_{\mathrm{K}^{-}}-\mathrm{m}_{\mathrm{K}}{ }^{+}\right), \text {glnV44,thi-1, deoR, gyrA96, recA1, } \\
\text { relA1, supE44, } \Delta(\operatorname{lac} Z Y A-\arg F) \cup 169, \lambda-,[\phi 80 d l a c \Delta(\operatorname{lac} Z) \text { M15]. }\end{array}$ \\
\hline KC8 (4) & $\begin{array}{l}\text { hsdR, leuB600, trpC9830, pyrF::Tn5, hisB463, lac } \Delta X 74 \text {, strA, galU, } \\
\text { galK. }\end{array}$ \\
\hline & Note: Tn5 confers kanr. \\
\hline $\mathrm{MG7} \alpha$ & 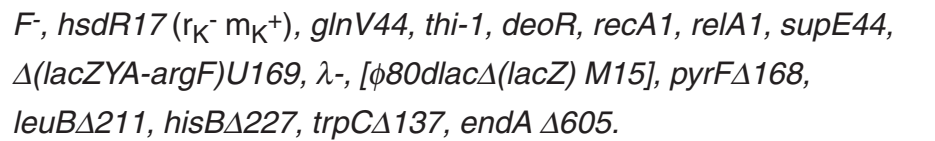 \\
\hline
\end{tabular}

vested and resuspended in Native Binding Buffer $(20 \mathrm{mM}$ phosphate, $500 \mathrm{mM}$ $\mathrm{NaCl}, \mathrm{pH} 7.8$ ), part of the ProBond ${ }^{\circledR}$ Purification System (Invitrogen, Carlsbad, CA, USA), containing protease inhibitor cocktail [1 mM 4-(2-aminoethyl)-benzene sulfonyl fluoride (AEBSF), $0.15 \mathrm{mM}$ pepstatin A, 0.15 $\mathrm{mM}$ E-64, $0.1 \mathrm{mM}$ bestatin, and $1 \mathrm{mM}$ EDTA]. The cell suspensions were treated with lysozyme $(100 \mu \mathrm{g} / \mathrm{mL})$ for $15 \mathrm{~min}$ and sonicated four times, with flash freezing in liquid nitrogen between each sonication. Finally, the lysate was cleared using a $0.8-\mu \mathrm{m}$ syringe filter. Lysates from different strains were normalized for protein content by the Bio-Rad Protein Assay Dye Reagent (Bio-Rad Laboratories) and used for assays of endA activity. All threitol (DTT)]. of the gene of interest.
endA assays were conducted in multicore buffer [25 mM Tris-acetate, $\mathrm{pH}$ $7.75,100 \mathrm{mM}$ potassium acetate, 10 $\mathrm{mM}$ magnesium acetate, $1 \mathrm{mM}$ dithio-

For each of the genes, $p y r F, \operatorname{leu} B$, $h i s B, \operatorname{trp} C$, and endA, the location of the gene in the $E$. coli chromosome was determined by a search of the National Center for Biotechnology Information's database (Bethesda, MD, USA) of the E. coli K-12 MG1655 complete genome (12). The location and orientation (+ or -) of each target gene was determined, and the sequence was saved for further analysis. These genome sequences were used to design primers that targeted a region of 100-300 bp in size, within $100 \mathrm{bp}$ from the start codon

\section{A.)}
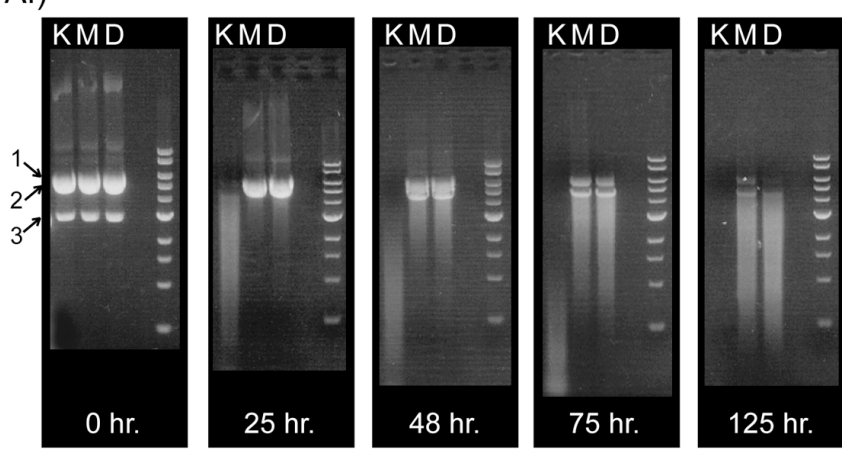

B.)

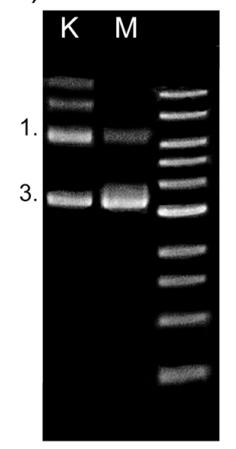

Figure 1. Assays of endA activity. (A) Qualitative assay of endA activity. The pUC9 plasmid DNA was treated with lysates from the strains $\mathrm{KC} 8(\mathrm{~K}), \mathrm{MG} 7 \alpha(\mathrm{M})$, and DH5 $\alpha(\mathrm{D})$. Supercoiled plasmid DNA was added to $E$. coli lysates and incubated at $37^{\circ} \mathrm{C}$. The samples were electrophoresed on $0.7 \%$ agarose gels, and the degradation of the plasmid DNA was observed by ethidium bromide staining after gel electrophoresis. (1) Open circular plasmid. (2) Linear plasmid. (3) Supercoiled plasmid. (B) Electrophoresis of QIAprep Spin Plasmid DNA. Plasmid preparations from KC8 (K) and MG7 $\alpha(\mathrm{M})$ cells were electrophoresed on a $0.7 \%$ agarose gel and observed by ethidium bromide staining.
For each target gene, homologous extensions were designed so that a recombination event would occur completely within the target gene and replace part of its sequence with the kanamycin resistance gene-disruption cassette. For each gene, 39-45 bp of homology were used and added onto the 20-bp of priming sequence for the template pKD4 (see Table 2 for all primer sequences).

Bacterial cells containing the plasmid pKD46 were grown in the presence of arabinose to induce the red recombinase system and stimulate homologous recombination (13).

DH $5 \alpha$ or its subsequent derivatives containing pKD46 were electroporated with the purified PCR-generated genedisruption cassettes. Kanamycin-resistant bacterial colonies were selected and tested for the disruption phenotype by streaking them onto M9 minimal media lacking uracil, leucine, tryptophan, or histidine. A single colony that exhibited the target mutation was then chosen and cured of pKD46. The strain was then used to generate new electrocompetent cells that were electroporated with pCP20 to facilitate the removal of the kanamycin marker as described earlier. Finally, the strain was cured of the second helper plasmid to yield a mutant strain with no residual antibiotic resistance, which allowed additional genes to be targeted for disruption.

DH5 $\alpha$ cells electroporated with a purified PCR product containing homologous regions for the $p y r F$ gene yielded several kanamycin-resistant colonies. When streaked onto M9 minimal medium that lacked uracil, some of these colonies did not produce any growth. One of these auxotrophic mutants was chosen for subsequent disruption. This new pyrF $\Delta 168$ deletion strain was prepared for the deletion of the $l e u B$ gene as described earlier. The process of gene disruption was repeated, and the $l e u B$, his $B$, and $\operatorname{trp} C$ genes were each disrupted and verified by testing on the appropriate minimal medium.

The pyrF $\Delta 168$, leuB $\Delta 211$, his $B \Delta-$ $227, \operatorname{trp} C \Delta 137$ deletion strain was then prepared for a disruption of the endA gene. The endA-PCR gene-disruption cassette was electroporated into these cells, yielding several hundred $\mathrm{kan}^{R}$ colonies. To test for the correct disrup- 
Table 2. Sequences of Amplification Primers

\begin{tabular}{|ll|}
\hline Primer Name & \multicolumn{1}{c|}{ Sequence } \\
\hline pyrF-pKD4 Fwd. & 5'-CGCTGTTACGAATTCTCCTGTGGTTGTTGCCCTTGAgtgtaggctggagctgcttc-3' \\
pyrF-pKD4 Rev. & 5'-GCAGCAGCGACAGCGTGCGCTGCAGTGTTGGGGATAcatatgaatatcctccttag-3' \\
leuB-pKD4 Fwd. & 5'-AACCGCTTTGCGATGCGCATCACCACCAGCCATTACGATgtgtaggctggagctgcttc-3' \\
leuB-pKD4 Rev. & 5'-GCAATGTCTGCACGCAGCGGACAGAATGCTTCCAGCCCCcatatgaatatcctccttag-3' \\
hisB-pKD4 Fwd. & 5'-ATTTTCAGGTGGACCGTTTTGATAAACTCGCCTTTGAACgtgtaggctggagctgcttc-3' \\
hisB-pKD4 Rev. & 5'-GCGATCCATCGCTTGCTCAGCCAGATAACGTTCCACCAGcatatgaatatcctccttag-3' \\
trpC-pKD4 Fwd. & 5'-TTTTAGCGAAAATCGTCGCAGACAAGGCGATTTGGGTAGgtgtaggctggagctgcttc-3' \\
trpC-pKD4 Rev. & 5'-GGCGGCAATGCGTGCTGGATCGAAATCATCACGGATCACcatatgaatatcctccttag-3' \\
endA-pKD4 Fwd. & 5'-ATGTACCGTTATTTGTCTATTGCTGCGGTGGTACTGAGCGCAGCAgtgtaggctggagctgcttc-3' \\
endA-pKD4 Rev. & 5'-GCCTGGCAAGCGCGTTGCACATACGGGTTATGATTGCCCTGCACCcatatgaatatcctccttag-3' \\
endA-Test Fwd. & 5'-tcgcctgcgggaaaactcgcccaggccaa-3' \\
endA-Test Rev. & 5'-caccaggcttccagatccatcgctggacgg-3' \\
Homologous extensions: sequences that target the gene of interest are uppercase. \\
$\begin{array}{l}\text { Sequences used for amplification of the knock-out cassette from the template plasmid, pKD4, or verification of the } \\
\text { endA disruption, are bold and lowercase. }\end{array}$
\end{tabular}

tion of the endA gene, we designed test primers to amplify the region flanking the end $A$ gene, and the disruption was confirmed by the size of the PCR products that were amplified (Table 2). Genomic DNA from several kanamycinresistant colonies was used to test for end $A$ gene disruption using this PCR assay. The end $A$ gene disruption was verified in several $\mathrm{kan}^{R}$ colonies. With the $\mathrm{kan}^{R}$ gene inserted into the endA gene, the PCR product was found to be the expected $2683 \mathrm{bp}$. After the $\mathrm{kan}^{R}$ marker was evicted, the product was found to be the expected $1296 \mathrm{bp}$ in size. These products were easily distinguished from the 1811-bp product expected for the parent strain DH5 $\alpha$. The resulting strain containing each of the deletion mutations described earlier was designated MG7 $\alpha$ (see Table 1 for genotype).

DNA degradation was observed qualitatively by treating samples of YEplac112 plasmid DNA (4.99 kb) with lysates from each $E$. coli strain (at $37^{\circ} \mathrm{C}$ ) for various time intervals and analyzing (approximately $500 \mathrm{ng}$ ) by gel electrophoresis. Figure 1A shows that the plasmid DNA that was treated with lysate from KC8 was dramatically degraded by $25 \mathrm{~h}$ and continued to degrade with further incubation (indicat- ed by the smear of low molecular weight DNA). The samples treated with DH5 $\alpha$ and MG7 $\alpha$ lysates showed some nicking after $25 \mathrm{~h}$. This is observed as a conversion of supercoiled plasmid to the open circular form. Further incubation converts the open circular form of the plasmid to the linear form and produces more degradation products in both the MG7 $\alpha$ and DH5 $\alpha$ samples. The MG7 $\alpha$ lysate appears to have less degradation potential when the 125-h time point is inspected.

The quality of QIAprep ${ }^{\circledR}$ spin plasmid DNA preparations (Qiagen, Mississauga, ON, Canada) that were produced by each strain was also tested. The YEplac112 plasmid DNA was prepared from $\mathrm{KC} 8$ and MG7 $\alpha$ cultures, and approximately $300 \mathrm{ng}$ were analyzed on a gel (Figure 1B). The KC8 lane shows a mixture of supercoiled and open circular plasmid, compared to the MG7 $\alpha$ sample, which is composed of supercoiled plasmid.

The quality of DNA produced by the strains MG7 $\alpha$ and KC8 was compared quantitatively by using the plasmid DNA for electroporation. Plasmid samples (YEplac112) extracted from MG7 $\alpha$ produced significantly more transformants than plasmid samples ex- tracted from $\mathrm{KC} 8(P<0.001)$. Twelve samples were prepared from each strain, and the MG7 $\alpha$ plasmid preparations produced on average three times as many transformants. This test was also conducted using cosmid DNA, and the effect was more pronounced. The MG7 $\alpha$ cosmid preparations produced 4.5 times more transformants than $\mathrm{KC} 8$ $(P<0.001)$.

MG7 $\alpha$ cells were transformed by electroporation (11) with comparable efficiencies to that of DH5 $\alpha$. MG7 $\alpha$ showed no growth on M9 drop-out media lacking uracil, leucine, histidine, or tryptophan. However, each of these mutations was successfully complemented by the URA3, LEU2, TRP1, and HIS3 yeast genes carried on the plasmids YEplac195, YEplac181, YEplac112, and pRS303, respectively. In addition, MG7 $\alpha$ has been successfully used to rescue numerous LEU2 plasmids from a two-hybrid screen conducted in our laboratory (R.D. Gietz, unpublished results).

We have produced a new E. coli strain, MG7 $\alpha$, with genetic markers equivalent to $\mathrm{KC} 8$ but superior plasmid DNA production characteristics. The strain MG7 $\alpha$ contains four auxotrophic mutations that make it ideally suited for 
the selection of the vectors that are used in two-hybrid strategies. MG7 $\alpha$ contains a deletion of the endA gene, compared to the point mutation in $\mathrm{DH} 5 \alpha$, which still allows for a residual level of endonuclease activity $(14,15)$. The end $A$ knock-out removes any residual end $A$ activity and thus allows MG7 $\alpha$ to produce higher quality plasmid DNA preparations than strains carrying end $A$ point mutations. In addition to its use in the yeast two-hybrid system, the qualities that it inherits from its parent strain, $\mathrm{DH} 5 \alpha$, and the end $A$ deletion we have introduced, make it a good all-purpose laboratory strain. This strain will be made available through ATCC (Reference no. MBA-78; Manassas, VA, USA).

\section{REFERENCES}

1.Bolivar, F., R.L. Rodriguez, P.J. Greene, M.C. Betlach, H.L. Heynecker, H.W. Boyer, J.H. Crosa, and S. Falkow. 1977. Construction and characterization of new cloning vehicles. II. A multipurpose cloning system. Gene 2:95-113.

2.Fields, S. and O. Song. 1989. A novel genetic system to detect protein-protein interactions. Nature 340:245-246.

3.Ratzkin, B. and J. Carbon. 1977. Functional expression of cloned yeast DNA in EScherichia coli. Proc. Natl. Acad. Sci. USA 74:487-491.

4.Struhl, K., D.T. Stinchcomb, S. Scherer, and R.W. Davis. 1979. High-frequency transformation of yeast: autonomous replication of hybrid DNA molecules. Proc. Natl. Acad. Sci. USA 76:1035-1039.

5.Datsenko, K.A. and B.L. Wanner. 2000. One-step inactivation of chromosomal genes in Escherichia coli K-12 using PCR products. Proc. Natl. Acad. Sci. USA 97:6640-6645.

6.Sambrook, J., E.F. Fritsch, and T. Maniatis. 1989. Molecular Cloning: A Laboratory Manual. CSH Laboratory Press, Cold Spring Harbor, NY.

7.Birnboim, H.C. and J. Doly. 1979. A rapid alkaline extraction procedure for screening recombinant plasmid DNA. Nucleic Acids Res. 7:1513-1523.

8.Girvitz, S.C., S. Bacchetti, A.J. Rainbow, and F.L. Graham. 1980. A rapid and efficient procedure for the purification of DNA from agarose gels. Anal. Biochem. 106:492-496.

9.Gietz, R.D. and A. Sugino. 1988. New yeastEscherichia coli shuttle vectors constructed with in vitro mutagenized yeast genes lacking six-base pair restriction sites. Gene 74:527534.

10.Sikorski, R.S. and P. Hieter. 1989. A system of shuttle vectors and yeast host strains designed for efficient manipulation of DNA in Saccharomyces cerevisiae. Genetics 122:1927.

11.Dower, W.J., J.F. Miller, and C.W. Ragsdale. 1988. High efficiency transformation of $E$. coli by high voltage electroporation. Nucleic Acids Res. 16:6127-6145.

12.Blattner, F.R., G. Plunkett, C.A. Bloch, N.T. Perna, V. Burland, M. Riley, J. ColladoVides, J.D. Glasner, et al. 1997. The complete genome sequence of Escherichia coli K-12. Science 277:1453-1474.

13.Murphy, K.C. 1998. Use of bacteriophage lambda recombination functions to promote gene replacement in Escherichia coli. J. Bacteriol. 180:2063-2071.

14.Cherepanov, P.P. and W. Wackernagel. 1995. Gene disruption in Escherichia coli: $\mathrm{TcR}$ and $\mathrm{KmR}$ cassettes with the option of Flpcatalyzed excision of the antibiotic-resistance determinant. Gene 158:9-14.

15.Durwald, H. and H. Hoffmann-Berling. 1968. Endonuclease I-deficient and ribonuclease I-deficient Escherichia coli mutants. J. Mol. Biol. 34:331-346.

16.Woodcock, D.M., P.J. Crowther, J. Doherty,
S. Jefferson, E. DeCruz, M. Noyer-Weidner, S.S. Smith, M.Z. Michael, et al. 1989. Quantitative evaluation of Escherichia coli host strains for tolerance to cytosine methylation in plasmid and phage recombinants. Nucleic Acids Res. 17:3469-3478.

Received 10 March 2003; accepted 16 May 2003.

Address correspondence to Daniel Gietz, Department of Biochemistry and Medical Genetics, University of Manitoba, Room P306, Pathology Building, 770 Bannatyne Avenue, Winnipeg, MB, Canada MR3E oW3.e-mail: gietz@cc.umanitoba.ca

\title{
Rapid isolation of Arabidopsis thaliana developing embryos
}

\author{
Sharyn E. Perry and Huai Wang \\ University of Kentucky, Lexington, $K Y, U S A$
}

BioTechniques 35:278-282 (August 2003)

Arabidopsis thaliana is a model plant for which many genetic and molecular tools are available. Another advantage of $A$. thaliana is its small size, allowing many plants to be grown in limited space. However, the diminutive size makes some experiments difficult. In particular, it is laborious to obtain enough isolated embryos for molecular or biochemical studies. Experiments involving developing embryos are further complicated because tissues containing $A$. thaliana embryos, such as siliques and seeds, can be difficult to work with because of secondary metabolites and polysaccharides. For example, we have found that RNA cannot be obtained from siliques or seeds using commercially available RNA isolation kits, such as RNeasy ${ }^{\circledR}$ (Qiagen, Valencia, CA, USA) or TRIzOL ${ }^{\circledR}$ reagent (Invitrogen, Carlsbad, CA, USA), methods that are widely used because they are fast and easy. Protocols to obtain RNA from these tissues are considerably more involved $(1,2)$.

Here we describe a simple method, based on density gradient centrifugation, to isolate relatively large quantities of $A$. thaliana embryos. Our method is faster and requires less handling of individual seeds and embryos than does manual dissection. Approximately $100 \mathrm{mg}$ torpedo to bent cotyledon stage embryos can be isolated in an afternoon. It is possible to isolate even younger embryos, as early as late heart stage. The embryos are suitable for a variety of experiments, including RNA isolation and protein extraction and analysis.

Developing siliques were opened and seeds removed into a few drops of $\mathrm{MC}$ buffer for protein work (3) (MC buffer is $10 \mathrm{mM}$ potassium phosphate, $\mathrm{pH}$ 7.0, $50 \mathrm{mM} \mathrm{NaCl}, 0.1 \mathrm{M}$ sucrose), or RNAlater ${ }^{\mathrm{TM}}$ (Qiagen) for RNA isolation, on a glass microscope slide. 